\title{
CORRESPONDENCE
}

\section{Assessing the Success of Masking in Acupuncture Trials: Further Insight}

\author{
Vance W. Berger
}

\section{KEYWORDS allocation concealment, Berger-Exner test, clinical trial, selection bias}

Editor: Hopton and MacPherson ${ }^{(1)}$ are correct in noting that ${ }^{(1)}$ attempts at masking are not always successful, hence ${ }^{(2)}$ unsubstantiated claims of masking are not convincing, hence ${ }^{(3)}$ guidance to cease assessing the success of masking should not be embraced. I take exception, however, to the authors calling this last point "controversial". I will concede that it is, in fact, controversial in the sense that tobacco was controversial at a time when certain medical groups still endorsed tobacco, or that the need for surgeons to wash their hands after procedures was controversial at a time when some surgeons simply did not wish to do this. In each case we have controversy arising from the pitting of valid, scientific concerns against special interest groups who, for whatever reason, choose to depart from logic. If we wish to confine our attention to science, then the controversy disappears, and what we have before us is guidance that is just plain wrong, and undeserving of the respect the authors have bestowed upon it.

Two other points are also worthy of mention. First, the authors noted a similarity in ${ }^{(1)}$ unmasking from efficacy and ${ }^{(2)}$ unmasking from side effects. In reality, these are not similar at all, other than that both involve unmasking. In the former case, we have unmasking arising from the fact that the treatment actually works. In other words, we have our answer, and if the observed efficacy was the cause of the unmasking, then it must have preceded the unmasking, so could not have been caused by unmasking. There is still the issue of unmasking causing exaggerated estimates of the effect of the treatment, and this is a problem too ${ }^{(2)}$. But at least we know that the null hypothesis cannot be true; the treatment works. In the latter case, we have no such assurance, so this is a more pressing concern.

Finally, unmasking leads to other biases besides the obvious ones. Specifically, when permuted blocks or other restricted randomization procedures are used, which is the case in almost all randomized trials, unmasking allows for prediction of future allocations, and this lack of allocation concealment allows patients to be selected strategically so as to create an advantage for one treatment group relative to the other ${ }^{(3,4)}$. This type of selection bias is not possible when masking is successful. Hence, a test for this type of selection bias serves also as a de facto test of the success of masking. As such, no evaluation of the success of masking can be complete without conducting and reporting the results of the Berger-Exner test, which is the definitive test of selection bias ${ }^{(4,5)}$.

This research received no specific grant from any funding agency in the public, commercial, or notfor-profit sectors.

\section{REFERENCES}

1. Hopton AK, MacPherson H. Assessing blinding in randomized controlled trials of acupuncture: challenges and recommendations. Chin J Integr Med 2011;17:173-176.

2. Berger VW. On the generation and ownership of alpha in medical studies. Control Clin Trials 2004;25:613-619.

3. Berger VW, Ivanova A, Deloria-Knoll M. Minimizing predictability while retaining balance through the use of less restrictive randomization procedures. Statistics Med 2003;22:3017-3028.

4. Berger VW. Selection bias and covariate imbalances in randomized clinical trials. John Wiley \& Sons, Chichester. 2005.

5. Berger VW, Exner DV. Detecting selection bias in randomized clinical trials. Control Clin Trials 1999;20:319-327.

(Received April 2, 2011) Edited by ZHANG Wen

(C)The Chinese Journal of Integrated Traditional and Western Medicine Press and Springer-Verlag Berlin Heidelberg 2011

Biometry Research Group, National Cancer Institute, Executive Plaza North, Suite 3131, 6130 Executive Boulevard, MSC 7354, Bethesda, MD 20892-7354

Tel: 301-435-5303, Fax: 301-402-0816, E-mail: vb78c@nih.gov DOI: $10.1007 / \mathrm{s} 11655-011-0790-3$ 